seems to be necessary: disease of vessels, sudden hyperæmia, or violence. In scurvy and small-pox, for instance, disease of vessels probably plays the most important part, as it does also in myelitis when complicated by hæmorrhage. Sudden hyperæmia appears to be the effective cause in those cases which arise in connexion with exposure to cold, extreme physical exertion, and sudden alterations of atmospheric pressure, such as occur in the case of divers. Finally, in cases such as the one I have taken for the subject of this lecture a strain or injury seems to produce the accident without causing any tangible alteration in the bones or spinal membranes, but in what manner it gives rise to hæmorrhage is not very clear. The case which I have now brought before your notice is the first I have met with in which simple primary hremorrhage of the cord has been shown by post-mortem examination to have the lesion which has proved fatal.

\section{ON BLOODLETTING.}

\section{BY SAMUEL WILKS, M.D., F.R.S.}

DR. OGLE having given you in an interesting communication his opinion on the value of bloodletting, I should in like manner be pleased to offer you my own experience, which is highly confirmatory both of his and that of Dr. Pye-Smith, Dr. Broadbent, and other eminent medical men. During the whole of my professional life I have occasionally used bloodletting as a remedial measure, and have probably adopted venesection in about thirty cases. In some lectures on Chest Diseases, published many years ago, for the use of students, I said, "As regards bleeding, there cannot be a doubt that in former times, from the truthful character of the records, its use was often attended with a strikingly good result. We read of persons sitting up in bed with great oppression of breathing, their faces gorged with blood, and the surgeon coming in with his lancet, bleeding in a full stream, and immediately quieting the respiratory process and relieving the circulation. In such cases there may have been pneumonia, bronchitis, or heart disease, but the results were nevertheless the same. This seems to show that in cases of great engorgement of the lungs or of the right side of the heart bleeding will afford relief, and therefore in similar circumstances I should advise you to bave recourse to the measure. When you find the venous system gorged in primary affections of the lungs, or in secondary congestions, as in heart disease, or from paralytic conditions, as in apoplexy, you cannot be wrong in bleeding; you relieve the venous system as well as the heart, and allow the circulating apparatus to right itself. The objection sometimes made that the patient is too weak, as indicated by the pulse, is futile, since, owing to the small amount of blood which reaches the left ventricle to be propelled onwards, the pulse is naturally small." Again, in my lectures on "Diseases of the Nervous System," I devote several pages to the consideration of bloodletting and its use when the lungs are gorged from paralysis of the chest or other cause. I may make the following quotation:-"Many of you saw the case of epilepsy which one of my clerks bled a few months ago. The man was a strong agricultural labourer, and came here suffering from severe epileptic fits. One afternoon, on going round, we were informed that he had never been out of a fit for four hours. We found him lying in bed with constant convulsive movements, but the most striking and alarming condition was the engorgement of the lungs and increasing lividity of the surface of the body. I requested that he should be bled, and one of you, wishing to distinguish himself at his first attempt, or from the lancet being over-sharp from disuse, fulfilled my object to the utmost ; the blood poured out in a torrent, the face rapidly became pale, the man opened his eyes and spoke for the first time since the morning, the interval having been a blank to him. The effect was the most striking I have ever witnessed, and it is worthy of note also that he had no fit for a long time afterwards."

It was very early in my student days that I was impressed with the value of bloodletting. I accompanied a general practitioner to see a little boy suffering from bronchitis after whooping-cough. He was lying half raised on a pillow gasping for breath, his face livid, eyes starting out of his head, and the superficial jugular making itself most appa- rent. The doctor, after gazing at this vein and feeling it, pulled out a lancet, and in spite of the protestations of the father opened it and let out several ounces of blood. The lividity passed away, the child sank back on the pillow in a tranquil sleep, and from that time speedily recovered. I have no records by me of the cases in which bleeding was adopted, but certain instances are deeply rooted in my memory. For example, a young woman was brought into the hospital in a dying state, gasping for breath, and livid; there was a mitral bruit, engorgement of the lung with hæmoptysis, albuminous scanty urine, and dropsy. We bled her from the arm, and the effect was immediate, the breathing became tranquil, lividity passed from the face, and improvement continued from that time. In cases of uræmic convulsions the effects of bleeding are very striking; the right heart is relieved, and poisoned blood is removed from the system. One case of this kind, indelibly fixed in my memory, is that of a neighbouring dressmaker, who, $I$ believe, is still alive. I was asked a few years ago to see this patient early one morning by her medical attendant as a satisfaction to him and her friends; she had been in convulsions all night, but he did not wish to disturb me before daybreak, believing the case to be hopeless. I found a middle aged woman in constant convulsive movements, froth issuing from her mouth, quite comatose, and almost pulseless. The whole body was livid and the extremities cold. The doctor said she was dying, and allowed me therefore to bleed her. I took away a small wash-hand basin of blood, and while flowing the lividity passed off, the convulsions ceased, the body gradually became warmer, and when I saw her in the middle of the day she was calm, and conversed with me. Her life was as clearly saved by the bleeding as if I had dragged her drowning out of the water; and this is more than I can say from the use of drugs. Dr. Ogle writes that little was said at the discussion about the use of leeches and cupping. I can remember a good example of treatment by each method. I was once asked to see a well.grown child a year old suffering from inflammation of the lungs. He was lying on his mother's lap, respiration being from 50 to 60 a minute, with deep episternal and hypogastric depression, inflated nostrils, lips blue, \&c. The medical man said he formerly should have leeched, but now waited for my sanction. I assented, and two leeches were applied. Believing that the child was too far gone to be relieved by any measures whatever, I was much surprised to be requested to see him again on the following day. I then found him breathing tranquilly, and comparatively well. The leeches had taken, and the bleeding had continued some time afterwards - in fact, much more than the medical man desired. It was, however, effectual

As regards cupping, I have seen it so often done in former years in hospital practice with advantage that $I$ cannot recall any individual cases, but the last case in private in which I saw cupping performed was in consultation with Mr. Parrott of Clapham shortly before his death. The patient was a middle-aged, stout woman, the subject of chronic bronchitis. She was sitting up in bed with great dyspncea, no expansion of lower part of lung, and considerable blueness of the face. Mr. Parrott said in former years he should have cupped her, and informed me that he had paid special fees to Mr. Hills of Guy's Hospital to learn the art, and he considered himself an adept. I willingly consented. He hastened home, brought his glasses, and applied them secundum artem (four in number) between the shoulders. He commented on his dexterity, and how he had removed the cups without leaving a drop of blood on the skin or the linen. We all stood in admiration at his adroitness, and gazed alternately at the relieved countenance of the patient and the satisfied expression of the doctor. Although she was not in extremis $\mathrm{I}$ believed the cupping saved her life, as she was daily growing worse.

I wish we had a more simple means of relieving the gorged portal system than incising the liver, as recommended by Dr. George Harley. Everyone must have seen the relief to the patient with cirrhosis after hremorrhage from the bowels. One case I shall never forget. A middle-aged woman, an inebriate, was when I was asked to see ber in an apparently dying condition. She had a very large liver, was covered with purpura spots, and was bleeding from the gums and mouth. The consultation was a mere formal one, and therefore I was much surprised to hear that she was still alive two days afterwards, and that I was requested to see her again. I did not recognise her, so altered was her appearance; her blotchy face had become pale, and while 
regarding her I was asked to look at a row of four chamber vessels arranged along the wall of the room. They were all full of dark blood. The melana saved her life, and she lived, I was told, some time afterwards. Besides the mere question of the value of bleeding in the mind of a medical man, there are two other considerations which influence him in his procedure. There is first his own personal reputation to be thought of, for just as the fashion of our forefathers was to "knock down" disease by every lowering measure, so the modern fashion is to "support" the patient. Consequently, if the latter died after bleeding, the doctor knew he would be blamed; but if he killed his patient by over-feeding, alcohol, or drugs, he would be thanked as having done his best. The other objection to bleeding which many men have is that they do not know how to do it; much less can they cup. I am sorry to say that I have usually had to perform the simple operation of venesection myself.

Grosvenor-street, W.

\section{A CASE OF GASTRO-ENTEROSTOMY FOR PYLORIC CANCER.}

BY A. W. MAYO ROBSON, F.R.C.S.,

HONORARY SURGEON TO THE LEEDS GENERAL INFIRMARY; PROFESSOR OF SURGERY IN THE YORKSHIRE COLLEGE; AND EXAMINER IN THE VICTORIA UNIVERSITY.

THE case is of interest as showing how safely and easily gastro-enterostomy can be performed, and how it is capable of prolonging life in cases unsuitable for the more radical operation of pylorectomy. In this instance the malignant disease had involved not only the pylorus, but the first and second parts of the duodenum, it being therefore far too extensive for removal. The case also illustrates the advantage of performing gastro-enterostomy by means of decalcified bone plates, since the operation can be performed very quickly. If this patient had had to submit to the old operation of gastro.enterostomy by means of a double row of sutures, I teel sure she would not have survived, as she was in an extremely exhausted state before being operated on. I would draw special attention also to the method em ployed in clamping the bowel, which I have found of great service in many operations of a similar nature, and which I first mentioned in connexion with my case of cholecystenterostiomy. I refer to the use of a simple elastic tourniquet in the shape of a piece of drainage-tube which is wound once round the isolated and emptied bowel, one knot being tied, and the two ends being seized with pressure forceps. So long as the tourniquet is on, the bowel is rendered bloodless, and there is not the slightest danger of the escape of gas or fæces. As soon as the necessary manipulations are efiected, the pressure forceps are released and the single knot at once unties itself. By this means much time is saved. The case is also of interest from the age of the patient, which was only twenty-seven; this led me to hope that the stricture might be of a simple nature.

Miss G-, aged twenty-seven, consulted my brother, Mr. Herbert J. Robson, on March 20th, 1890, on account of a tumour in the epigastrium, accompanied by pain, sickness, and vomiting, the pain and sickness being usually worse after food, but coming on at times quite independently of diet. She stated that she had been well up to the middie of February, when she began to have shooting pains in the region of the stomach, lasting usually about half an hour, and coming on at intervals of two or three hours. This continued for three weeks, when the tumour was noticed in the epigastrinm, and on the appearance of the tumour the pain became somewhat relieved, but at that time the nausea and vomiting began. Exertion caused an aggravation of the symptoms. From the commencement of her illness up to the time of being seen she had gradually lost fiesh. The tumour was situated in the middle line, mid way between the umbilicus and ensiform cartilage; it was about the size of a small orange, tender on pressure, hard, nodular, and freely movable; it rose and fell with respiration, and had communicated to it the pulsation of the aorta. On April 3rd she had further lost llesh, and complained of persistent vomiting, with occasional offensive eructations. On the 10th I eaw her for the first time with my brother, and we advised her to go into the infirmary for operation. On admission to the infirmary on April 17th her symptoms still continued, but it was noticed that the epigastric ${ }^{\circ} \mathrm{s}$ welling became at times hard and prominent and afterwards flattened out, then being less distinct. On some occasions the hardness extended to the whole of the stomach, the shape of which could then be clearly seen through the abdominal walls. The following report was given by Dr. Light, resident medical offeer:- "Vomiter matter, reaction acid, free hydrochloric acid present no sarcinæ found. Signs of consolidation in the right apex of the lung." On April 29th a note was made to the effect that the patient was vomiting several times each day. On several occasions splashing sounds were heard on percussing the abdomen from side to side. On April 30th, under the influence of ether the abdomen baving been thoroughly cleansed and asepti. cised, and the stomach previously washed out with 8 solution of boro-glyceride, an incision was made in the middle line from about two inches below the sternum to the umbilicus. The peritoneum having been opened, the stomach at once came into view. It was then found that the swelling occupied the pyloric end of the stomach and the first and second parts of the duodenum, so that pylorectomy was quite out of the question. It was therefore determined to perform gastro-enterostomy. The omentum was raised and the attached part of the jejunum easily found and traced downwards for $a$ few inches; a loop of the bowel was then brought forward by the right finger and thumb and emptied of its contents by the pressure of the left finger and thumb, and then encircled by a thin piece of elastic tubing, which was tied once and then seized with pressure forceps. The bowel was not only emptied of its contents, but was rendered bloodless, so that when it was opened at a convenient spot of its anterior surface, by an incision in its long axis suffi. ciently large to admit the decalcified bone plate, there was neither fear of extravasation nor of bleeding. A similar plate was introduced into the stomach. The upper and lower stitches of the two plates were made to pierce the walls of the stomach and bowel respectively, the right and left stitches being brought out through the openings in the stomach and bowel. On tying the plates together the jejunum and stomach seemed to be closely applied; but in order to render the parts more secure, six Lembert's sutures of thin silk were used to stitch the viscera together, just external to the bone plates. In order to prevent the bowel kinking, an extra suture fixed it to the stomach wall about an inch from the edge of the plate. No soiling of the peritoneum oceurred throughout the operation, and the external wound was closed in the usual way with silk sutures. After the operation the patient complained of a little pain, and vomited a quantity of dark-coloured fluid which contained blood. On May 3rd the patient vomited twice, but there was no blood in the vomit, which, however, smelt slightly offensive, as if there might be some regurgitation of bowel contents through the artificial opening. The patient had no pain. On the 4th she still felt sick, but vomiting was controlled by half a grain of morphia given in the morning. She had nutrient injections thrice daily, and was able to take a little champagne and milk and soda-water by the mouth. On the 7 th a note was made that the patient was improving every day. On the 8 th there was no sickness; the wound was dressed and found to be healed by first intention. All the sutures were removed. The temperature had never been above the normal. She could then take a cup of tea and milk and soda-water by the mouth. On the 15th she could take oysters, bread-and-butter, and tea. On the 17th there was a little vomiting; but on the 19th she was able to take solid food and did not vomit. The patient was allowed to get up on the 26 th. On the 29 th her weight was only 5 st. $41 \mathrm{~b}$, , although she was taking more food and only vomited occasionally, not more than once in the twenty-four hours. On June 5th she had gained a pound in weight during the week, and on the loth a note was made that the general condition was improving, although there was a little vomiting. She was sent to the Ida Convalescent Home. On July 8 th the following note was made by $\mathrm{Mr}$. Fudson, the resident medical officer of that institution:- "Since the patient's admission to the Ida Home she has made no progress. Has vomited once or twice daily, and has lost weight. She has got up every day and has been able to take light diet, chiefly of a liquid character, but also solid food, such as bread.andbutter, fisb, \&c. Besides the nourishment of which she has 